\title{
Characteristics of high altitude oxygen ion energization and outflow as observed by Cluster: a statistical study
}

\author{
H. Nilsson ${ }^{1}$, M. Waara ${ }^{1}$, S. Arvelius ${ }^{1}$, O. Marghitu ${ }^{2,3}$, M. Bouhram ${ }^{2, *}$, Y. Hobara ${ }^{1,{ }^{* *}}$, M. Yamauchi ${ }^{1}$, R. Lundin ${ }^{1}$, \\ H. Rème ${ }^{4}$, J.-A. Sauvaud ${ }^{4}$, I. Dandouras ${ }^{4}$, A. Balogh ${ }^{5}$, L. M. Kistler ${ }^{6}$, B. Klecker ${ }^{2}$, C. W. Carlson ${ }^{7}$, \\ M. B. Bavassano-Cattaneo ${ }^{8}$, and A. Korth ${ }^{9}$ \\ ${ }^{1}$ Swedish Institute of Space Physics, Kiruna, Sweden \\ ${ }^{2}$ Max-Planck-Institut für Extraterrestriche Physik, Garching, Germany \\ ${ }^{3}$ Institute for Space Sciences, Bucharest, Romania \\ ${ }^{4}$ Centre d'Etude Spatiale des Rayonnements, Toulouse, France \\ ${ }^{5}$ Imperial College of Science, Technology and Medicine, London, UK \\ ${ }^{6}$ University of New Hampshire, Durham, USA \\ ${ }^{7}$ Space Science Laboratory, University of California, Berkeley, USA \\ ${ }^{8}$ Istituto di Fisica dello Spazio Interplanetario, Roma, Italy \\ ${ }^{9}$ Max-Planck-Institut für Sonnensystemforschung, Katlenburg-Lindau, Germany \\ *now at: CETP-CNRS, Saint-Maur, France \\ ** now at: University of Sheffield, Sheffield, UK
}

Received: 12 December 2005 - Revised: 6 March 2006 - Accepted: 8 March 2006 - Published: 19 May 2006

\begin{abstract}
The results of a statistical study of oxygen ion outflow using Cluster data obtained at high altitude above the polar cap is reported. Moment data for both hydrogen ions $\left(\mathrm{H}^{+}\right)$and oxygen ions $\left(\mathrm{O}^{+}\right)$from 3 years $(2001-2003)$ of spring orbits (January to May) have been used. The altitudes covered were mainly in the range $5-12 R_{E}$ geocentric distance. It was found that $\mathrm{O}^{+}$is significantly transversely energized at high altitudes, indicated both by high perpendicular temperatures for low magnetic field values as well as by a tendency towards higher perpendicular than parallel temperature distributions for the highest observed temperatures. The $\mathrm{O}^{+}$parallel bulk velocity increases with altitude in particular for the lowest observed altitude intervals. $\mathrm{O}^{+}$ parallel bulk velocities in excess of $60 \mathrm{~km} \mathrm{~s}^{-1}$ were found mainly at higher altitudes corresponding to magnetic field strengths of less than $100 \mathrm{nT}$. For the highest observed parallel bulk velocities of $\mathrm{O}^{+}$the thermal velocity exceeds the bulk velocity, indicating that the beam-like character of the distribution is lost. The parallel bulk velocity of the $\mathrm{H}^{+}$and $\mathrm{O}^{+}$was found to typically be close to the same throughout the observation interval when the $\mathrm{H}^{+}$bulk velocity was calculated for all pitch-angles. When the $\mathrm{H}^{+}$bulk velocity was calculated for upward moving particles only the $\mathrm{H}^{+}$parallel bulk velocity was typically higher than that of $\mathrm{O}^{+}$. The parallel bulk velocity is close to the same for a wide range of
\end{abstract}

Correspondence to: H. Nilsson

(hans.nilsson@irf.se) relative abundance of the two ion species, including when the $\mathrm{O}^{+}$ions dominates. The thermal velocity of $\mathrm{O}^{+}$was always well below that of $\mathrm{H}^{+}$. Thus perpendicular energization that is more effective for $\mathrm{O}^{+}$takes place, but this is not enough to explain the close to similar parallel velocities. Further parallel acceleration must occur. The results presented constrain the models of perpendicular heating and parallel acceleration. In particular centrifugal acceleration of the outflowing ions, which may provide the same parallel velocity increase to the two ion species and a two-stream interaction are discussed in the context of the measurements.

Keywords. Magnetospheric physics (Magnetopause, cusp and boundary layers; Magnetosphere-ionosphere interactions; Solar wind-magnetosphere interactions)

\section{Introduction}

The circulation and energization of ionospheric origin oxygen ions play a fundamental role in the dynamics of the earth's magnetosphere (e.g. Shelley et al., 1972; Winglee et al., 2002). The non-thermal energization and outflow of planetary origin ions may also be an important lossmechanism for planetary atmospheres as discussed by for example Lundin et al. (1989); Seki et al. (2001). Understanding the full circulation of oxygen ions in the earth's magnetosphere requires an understanding of all steps from the

Published by Copernicus GmbH on behalf of the European Geosciences Union. 
initial upflow in the ionosphere, subsequent energization and outflow along the magnetic field-lines and finally the transport in the tail to the inner magnetosphere or loss through the magnetopause.

The initial ionospheric upflow, usually associated with strong electric fields and associated Joule heating can be studied by incoherent scatter radar (e.g. Nilsson et al., 1996; Ogawa et al., 2003). These upflows are gravitationally bound and may result in later downflows also observable by incoherent scatter radars. However, in the topside auroral ionosphere significant transverse heating is often observed (e.g. Norqvist et al., 1998). Such low altitude upflow and energization are likely to determine the fluxes of all outflow also at higher altitude as it represents a modulation of the source. The transversely accelerated ions subsequently move outward, their transverse energy gradually transformed to parallel energy by the mirror force. Such transversely heated and subsequently outflowing ions observed close to the heating region are known as "conics" (for recent reviews see André and Yau, 1997; Yau and André, 1997; Moore et al., 1999). At larger distances from the source the conic shape cannot be resolved and the ions are observed as "beams". A beamlike distribution may also be caused by upward acceleration in an upward-directed field-aligned potential drop which frequently occur in the auroral zone. A two-stage acceleration involving first transverse heating at low altitudes followed by field-aligned acceleration has for exampled been suggested by Klumpar et al. (1984).

The study presented here is a follow up of a case study on $\mathrm{O}^{+}$outflow at high altitude over the polar cap reported by Nilsson et al. (2004) (paper 1). It thus constitutes a study of the link between the low (e.g. Norqvist et al., 1998; Andersson et al., 2005) and mid altitude energization related to the cusp region (e.g. Bogdanova et al., 2004) and the tail $\mathrm{O}^{+}$ beams reported by Frank et al. (1977); Seki et al. (1998).

$\mathrm{O}^{+}$particles outflowing over the polar cap originate from the cusp region as has been shown by Dubouloz et al. (2001); Bouhram et al. (2003, 2004); Nilsson et al. (2004); Bogdanova et al. (2004). Paper 1 reported both increasing parallel velocity and increasing isotropic temperature of the $\mathrm{O}^{+}$ beam with altitude. The temperature estimates in paper 1 were of the kinetic temperature, i.e. an integration of observed particles, not a fit of a Maxwellian. The observed distribution of $\mathrm{O}^{+}$parallel bulk velocity could be explained by transverse heating at around $1.5 R_{E}$ altitude in combination with the velocity filter effect and observed convection. However this explanation was not consistent with observed perpendicular temperatures and the parallel to perpendicular temperature ratios (which were isotropic). Gradual (though not necessarily continuous) heating over extended altitude intervals up to at least close to the observation altitude was suggested as a plausible explanation of the observations. The questions which arose out of the case study but which could not be answered with confidence based on just one case were:
(1) In paper 1 an apparent relation between the fieldaligned bulk velocity of $\mathrm{H}^{+}$and $\mathrm{O}^{+}$was reported. Is this a general result for outflow over the polar cap?

Such a coupling between the two ion species has been reported previously, mainly in the tail (Frank et al., 1977; Seki et al., 1998) but also at lower altitudes in the auroral zone (e.g. Möbius et al., 1998, and references therein). For the particular case of paper 1 the $\mathrm{O}^{+}$ions had a higher parallel bulk velocity than $\mathrm{H}^{+}$, but when the latter was calculated for upward moving particles only the velocity was close to the same. This indicates that an energization mechanism which is more efficient for heavy ions (Antonova, 1983; Guglielmi and Lundin, 2001; Cladis, 1986) and / or a two-stream instability between the two ion species (Bergmann et al., 1988; Ludlow and Kaufman, 1989; Winglee et al., 1993; Krauklis et al., 2001) is operating. In this paper we present a statistical investigation of the relation between $\mathrm{H}^{+}$and $\mathrm{O}^{+}$bulk parallel velocities as observed over the polar cap.

(2) Do further heating / cceleration of $\mathrm{O}^{+}$ions generally take place at high altitude $\left(5-10 R_{E}\right)$ ?

Attempts to relate the energy of observed outflowing oxygen ions in the cusp region to observation altitude clearly demonstrate that the energy increase with altitude at least in the lower altitude intervals up to $2 R_{E}$ (Dubouloz et al., 2001). Bouhram et al. (2004) reported a saturation of the $\mathrm{O}^{+}$ heating rates above $4.5 R_{E}$. Work based on Polar (Lennartsson et al., 2004) showed that the $\mathrm{O}^{+}$energy is significantly increased at 4-9 $R_{E}$ as compared to perigee passes at $2 R_{E}$ but no subdivision of the higher altitude region was performed. This was done by Arvelius et al. (2005) (paper 2) who surveyed peak differential particle flux and energy of peak differential particle flux for all $\mathrm{O}^{+}$ion beam events observed over the polar cap by Cluster during January to May from 2001 to 2003. They demonstrated that the statistical distribution of more energetic ions ( $1 \mathrm{keV}$ and above) in the 6-12 $R_{E}$ geocentric distance interval was not consistent with just a velocity filter effect in combination with a low altitude heating source. Additional energization at high altitude (8$12 R_{E}$ geocentric distance) was clearly evident. The nature of this further heating / acceleration could not be identified based on the used data set. The first question has thus already been answered, but the precise nature of this high altitude energization remains poorly understood. We use moment data of both $\mathrm{O}^{+}$and $\mathrm{H}^{+}$to shed more light on the high altitude energization of $\mathrm{O}^{+}$ions. In particular the perpendicular ion temperature is an important complement to paper 2, as high perpendicular temperatures at high altitudes are inconsistent with passive transport due to the action of the mirror-force.

(3) In paper 1 the ion temperatures reported showed a tendency towards isotropy (though with large deviations for single measurement points). What causes the tendency towards isotropic temperature of the $\mathrm{O}^{+}$beam observed at high altitude, and is it a general result? 
Simple calculations and simulations performed in paper 1 showed that if the upper magnetosphere is a passive transport region the observed kinetic temperature of the ion beam should not be isotropic. The perpendicular temperature is continuously decreased by the action of the mirror force, and both the parallel and perpendicular range of particle velocities observed (i.e. the kinetic temperature) is determined by the time of flight dispersion, i.e. higher temperatures for a shorter time-of-flight from the source. We follow up paper 1 with a study of how temperature ratios and the perpendicular temperature varies with altitude. A more complete study of the case reported in paper 1 has now been performed, involving also wave data and simulations (Hobara et al., 2005, paper 3$)^{1}$. The statistical data is discussed in the light of this more complete case study.

\section{Observations}

\subsection{Observational Techniques}

We use data from the Cluster Ion Spectrometer (CIS) onboard Cluster II spacecraft 4 . The CIS instrument is described in detail in Rème et al. (2001). CIS consists of two different ion spectrometers, Composition Distribution Function (CODIF) which can resolve the major magnetospheric ions and Hot Ion Analyzer (HIA) which has no mass resolution but higher angular and energy resolution. We will only present results from the CODIF instrument.

CODIF can resolve $\mathrm{H}^{+}, \mathrm{He}^{++}, \mathrm{He}^{+}$and $\mathrm{O}^{+}$through a time-of-flight technique. The detector has a field-of-view of $360^{\circ}$ orthogonal to the spin plane, divided into 16 sectors of $22.5^{\circ}$ each. The angular resolution is likewise $22.5^{\circ}$ in the spin plane. The energy coverage in the modes of interest to us is from $15 \mathrm{eV}$ per charge up to $38 \mathrm{keV}$ per charge in up to 30 logarithmically spaced steps with a $\Delta E / E$ of 0.16 .

Furthermore we use data from the Cluster fluxgate magnetometers (Balogh et al., 2001).

\subsection{Data set}

The data set consists of all $\mathrm{O}^{+}$beams clearly seen in the energy spectrograms of the CODIF data for spring (January to May) of the years 2001 to 2003. This corresponds to high altitude passes over the polar cap. Most of the ion beams were observed at geocentric distances between 5 and $12 R_{E}$. The beams were identified through visual inspection of energy spectrograms. A clear and typical example is shown in Fig. 1. Only beam events lasting more than an hour were included. Thus other types of more dynamically occurring

\footnotetext{
${ }^{1}$ Hobara, Y., Nilsson, H., Arvelius, S., Lundin, R., Sundkvist, D., André, M., Cornilleau-Wehrlin, N., Balogh, A., Buchert, S. C., Rème, H., and Sauvaud, J.-A.: Cluster observations of high altitude oxygen outflow burst and associated wave activities, Ann. Geophys, in preparation, 2006.
}

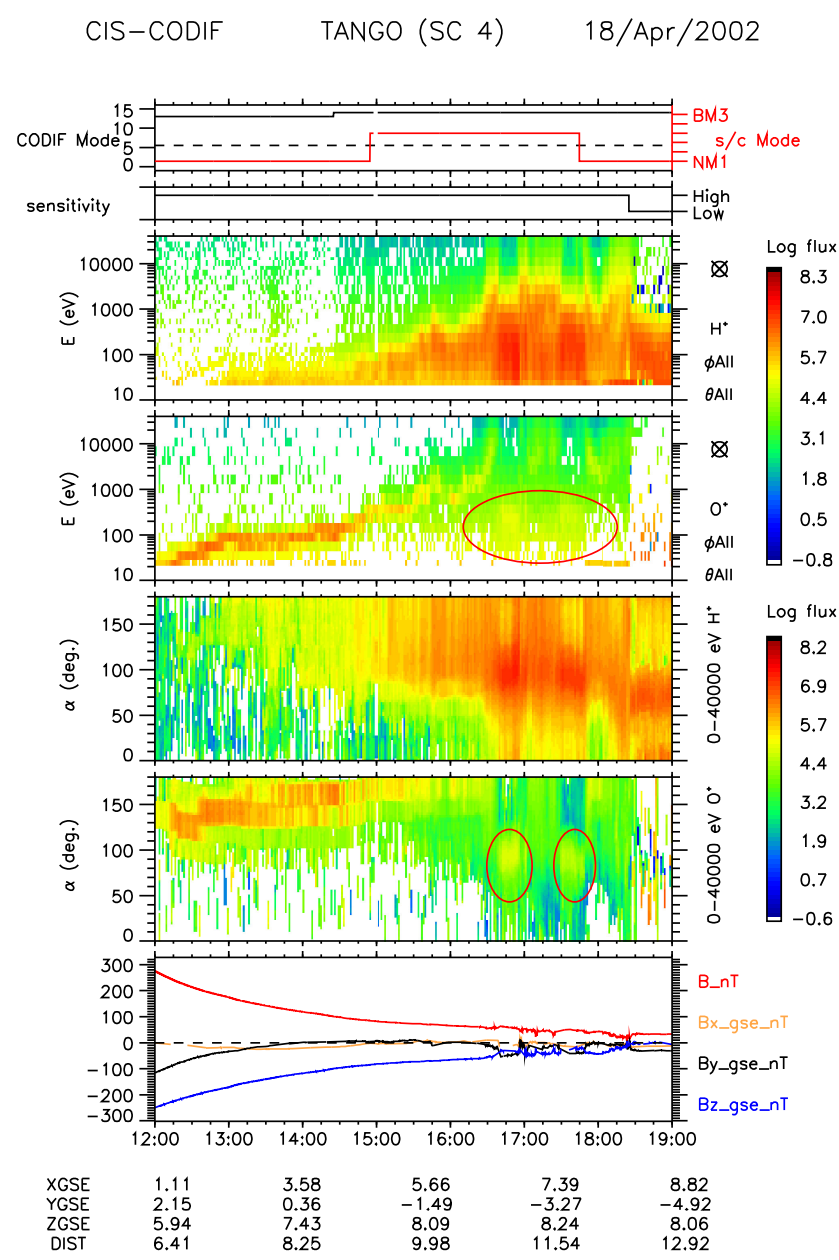

Fig. 1. Cluster spacecraft 4 data from 20020418. Panel 1 shows spacecraft and instrument mode, panel 2 instrument sensitivity. Panels 3 and 4 show the $\mathrm{H}^{+}$and $\mathrm{O}^{+}$energy spectrograms, particle flux integrated over all directions. A part of the $\mathrm{O}^{+}$spectrogram contaminated by $\mathrm{H}^{+}$is indicated with a red circle. Panels 5 and 6 shows pitch-angle spectrograms of $\mathrm{H}^{+}$and $\mathrm{O}^{+}$respectively. The angles are in the spacecraft reference frame so the apparent pitch angle for the cold $\mathrm{O}^{+}$beam is actually the angle between the parallel velocity and convection velocity. Pitch-angle 0 is along the direction of the magnetic field so that $180^{\circ}$ is outflow in the northern hemisphere. Panel 7 shows the magnetic field, both total (red line) and GSE components (orange, black and blue for $\mathrm{x}, \mathrm{y}$ and $\mathrm{z}$ respectively).

beams are not included in this work (e.g. Kistler et al., 2002). Characteristic features of the energy spectrograms are the relatively narrow structure in the $\mathrm{O}^{+}$spectrogram distribution, the continuous increase of energy in the higher altitude part of the orbit (which also maps to lower geomagnetic latitude) for both species, and the general similarity between the $\mathrm{O}^{+}$ beam and the high-energy cut-off of the $\mathrm{H}^{+}$flux distribution. The intense $\mathrm{H}^{+}$fluxes are clearly of magnetosheath origin (see paper 1 and papers by Bouhram et al. (2003); Bogdanova 


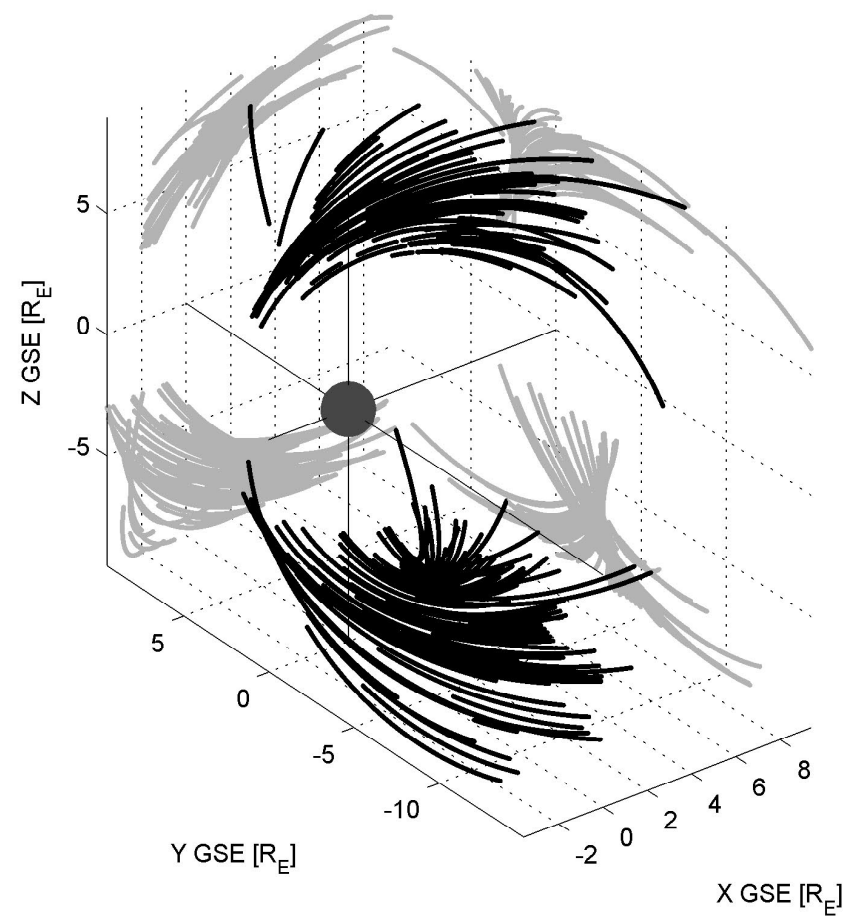

Fig. 2. GSE coordinates of all valid data points used in the study (black dots). The points usually forms continuous lines (individual orbits). The projection on the $\mathrm{X}-\mathrm{Z}$ and $\mathrm{Y}-\mathrm{Z}$ plane is shown with grey points.

et al. (2004) for further discussions on this). The low energy $\mathrm{H}^{+}$fluxes seen at the highest latitudes may in principle be of ionospheric origin but they may also be of magnetosheath origin, the low energy resulting from time-of-flight effects. As the large fluxes of $\mathrm{H}^{+}$are of magnetosheath origin we will in the discussion treat all $\mathrm{H}^{+}$as being of magnetosheath origin.

The characteristic feature of the $\mathrm{O}^{+}$pitch-angle distribution is the narrow distribution (the angular resolution of the instrument can be seen in Fig. 1 panel 6 as the width of the region with significant flux observed between 12:00 and 16:00 UT). Because the thermal velocity for $\mathrm{O}^{+}$is lower than the drift velocities (it is "cold") the apparent pitch-angle of the distribution is actually the angle between the parallel bulk velocity and the perpendicular bulk velocity (the convection). This is because the pitch-angle is calculated in the spacecraft reference frame and for the relatively cold $\mathrm{O}^{+}$this makes a significant difference. After 16:00 UT the $\mathrm{O}^{+}$population is less beam-like and hotter than the $\mathrm{H}^{+}$population. The energy spectrogram may still look thinner for $\mathrm{O}^{+}$than for $\mathrm{H}^{+}$ but this is because of the higher bulk drift energy in combination with the logarithmic y-scale. The statistics were calculated for all three spacecraft with working CODIF instruments $(1,3$, and 4$)$ though we will use only data from spacecraft 4 in this report. Moments were calculated for energies above $40 \mathrm{eV}$ to avoid the problems that sometimes occur in the lowest energy channels of CODIF. The CCAT software was used to calculate the moments from the 3-D distributions transmitted to ground. Measurements of the magnetic field were used in the calculation of parallel and perpendicular temperatures (not the diagonalization method sometimes used). The temperature is thus a kinetic temperature calculated by numerical integration of the measured particle distribution. The data set consists of 120 events and about 350000 data points of $16 \mathrm{~s}$. ( 4 spin accumulation) moment data. This was further reduced to 34000 datapoints of 1-min resolution where both $\mathrm{H}^{+}$and $\mathrm{O}^{+}$data were present and valid. The initial validation consisted of a check that the perpendicular ion temperature estimate was non-zero and that the absolute value of the GSE $\mathrm{z}$ coordinate was larger than 3 . Data with an $\mathrm{O}^{+}$field-aligned velocity of less than $22 \mathrm{~km} \mathrm{~s}^{-1}$ was excluded, as this corresponds to the minimum used $\mathrm{O}^{+}$energy (for the cold beam of Fig. 1 this corresponds to the lowest energy part of the beam observed just after 12:00 UT). Further data validation is discussed in Sect. 3. The same time periods were used in paper 2, but then only peak differential flux and energy of peak differential flux were used, not the moment data used in this study. Figure 2 shows the GSE coordinates of all valid data points (black) and the projection of the location of the data points on the $\mathrm{X}-\mathrm{Z}$ and $\mathrm{X}-\mathrm{Y}$ planes (grey).

\section{Data validation}

The CODIF moment data used in this study may suffer from two important limitations which must be followed up carefully: Contamination of the $\mathrm{O}^{+}$mass channel from high fluxes of $\mathrm{H}^{+}$and incorrect moment estimates due to the limited resolution of the instrument. The polar cap $\mathrm{O}^{+}$beams represent a challenge in both cases as will be discussed in the following sections.

\subsection{Contamination of the $\mathrm{O}^{+}$mass channel}

The CODIF instrument achieves mass separation through a time-of-flight system which is in principle very good at separating different masses (or more specifically mass per charge). In spite of this, for intense fluxes of $\mathrm{H}^{+}$other mass channels may be affected by false counts (an example is indicated with a red circle in the energy spectrogram of $\mathrm{O}^{+}$ shown in Fig. 1). This is due to chance start-stop coincidences caused by high proton count rates. For intense enough fluxes of $\mathrm{H}^{+}$these chance coincidences will produce significant background counts also in the $\mathrm{O}^{+}$time-of-flight range. This can be checked a posteriori most of the time, because a data product containing the time-of-flight histogram (product 28, see Rème et al. (2001)) is often transmitted to ground for at least one spacecraft at a time. One may then check if the $\mathrm{O}^{+}$counts correspond to a local maximum at the corresponding times in the time-of-flight histogram. We have done this 
for a number of cases, for example the case 2001-04-12 discussed in paper 1 , but it is not feasible to use this technique in a statistical study. Instead we have utilized the fact that the $\boldsymbol{E} \times \boldsymbol{B}$ drift should dominate perpendicular drift and be the same for all ion species in the energy range and location studied (below $40 \mathrm{keV}$ in the polar cap). For the case when the $\mathrm{O}^{+}$counts are all false counts caused by the intense $\mathrm{H}^{+}$fluxes seen simultaneously the estimate of the $\mathrm{O}^{+} \boldsymbol{E} \times \boldsymbol{B}$ drift will be $1 / 4$ of the estimate of the $\mathrm{H}^{+} \boldsymbol{E} \times \boldsymbol{B}$ drift. This is because the false $\mathrm{O}^{+}$counts appear in the same energy channels as the real $\mathrm{H}^{+}$counts (though at much lower intensity). When these counts are interpreted as $\mathrm{O}^{+}$all velocity moments are one-fourth of the corresponding $\mathrm{H}^{+}$velocity moments (the difference in velocity for the same energy being the square root of the mass ratio which is 16). The data set indeed shows a clear peak in the distribution of $\mathrm{O}^{+}$vs. $\mathrm{H}^{+} \boldsymbol{E} \times \boldsymbol{B}$ drift at the one-to-four ratio (not shown). Because of limited angular and energy resolution (mostly affecting the cold $\mathrm{O}^{+}$population, see next section) one cannot demand that the $\mathrm{O}^{+}$to $\mathrm{H}^{+} \boldsymbol{E} \times \boldsymbol{B}$ ratio should be close to unity for uncontaminated data, there will be considerable scatter. Empirical tests indicate that demanding the ratio to be above 0.5 is suitable. This test removes a significant amount of datapoints, the remaining points are approximately 15000 . Interestingly enough, the removal affects mainly the $\mathrm{O}^{+} / \mathrm{H}^{+}$ parallel velocity ratio distribution. Most of the other statistics shown in this paper looked very similar when the $\mathrm{H}^{+}$ contaminated data was included. This is because high fluxes of magnetosheath ions are mainly seen in regions where the $\mathrm{O}^{+}$population is hotter than the $\mathrm{H}^{+}$population so that the contamination does not affect the temperature so much.

\subsection{Effects of limited angular and energy resolution}

The $\mathrm{O}^{+}$ion beams observed over the polar cap are often cold. With this we mean that the width of the energy spectrum (the temperature) is small compared to the bulk drift velocity which is mainly along the field-line. This means that the field-aligned bulk velocity is well estimated but somewhat limited in accuracy by the logarithmic steps of the energy sampling (which reflects the actual energy resolution of the instrument). The logarithmic stepping of the energy sampling also limits the accuracy of the temperature estimates. The temperature must be high enough to cover several energy sample intervals for a decent temperature estimate. The dominance of the bulk velocity may also mean that the beam is seen only in one sector $\left(22.5^{\circ}\right.$ resolution) of the instrument. This may lead the moment computation to attribute $\tan 22.5^{\circ} / 2$ or $20 \%$ of the bulk speed to thermal speed or set it to zero, depending on how the algorithm to determine temperature is implemented. These limitations are discussed in Paschmann and Daly (1998). When only one sector detects the particles our analysis sets the temperature to zero, and this data is removed from our data set in the initial validation. For an estimate of how important these limitations are
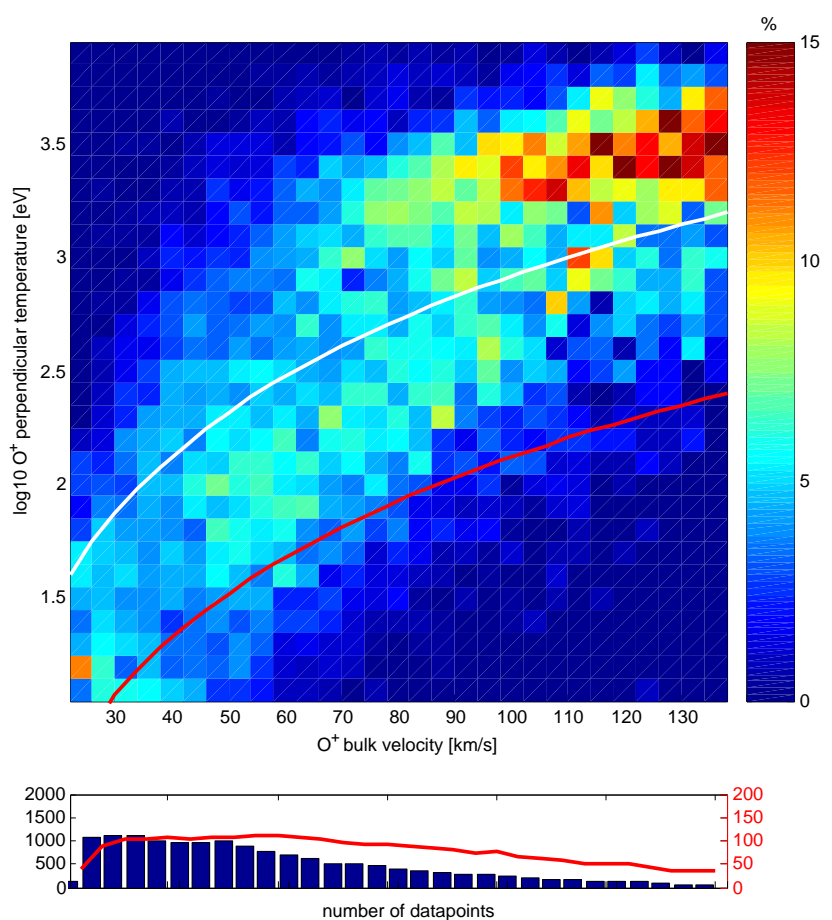

Fig. 3. Upper panel: Distribution of perpendicular $\mathrm{O}^{+}$temperature [log eV] for each interval of $\mathrm{O}^{+}$bulk velocity $\left[\mathrm{km} \mathrm{s}^{-1}\right]$. Each column is normalized, the sum of all data bins in a column is $100 \%$. Also shown are the line of equal thermal and bulk velocity (white) and the line of $16 \%$ thermal to bulk energy ratio (red). Lower panel: number of data points contributing to each column (blue bars, left $y$-axis), and the number of measurement days contributing to the column (red line, right y-axis).

for our data we present a plot in Fig. 3 of the distribution of perpendicular $\mathrm{O}^{+}$temperature for each interval of $\mathrm{O}^{+}$bulk velocity. Each column is normalized, the sum of all data bins in a column is $100 \%$. The lower panel of the figure shows the number of data points contributing to each column (blue bars, left y-axis), and the number of measurement days contributing to the column (red line, right y-axis). Also shown are two lines, one showing the equal thermal and bulk velocity (white line) and one showing the $40 \%$ thermal to bulk velocity ratio (red line, corresponding to the energy resolution of $16 \%$ of the instrument). Measurements closer to the white line provide good estimates of temperatures, whereas those closer to the red line will suffer from limited resolution effects. Most of the actual samples fall in the region between the red line (energy resolution limit) and the upper white line (equal thermal and bulk velocity energy). Measurements well below the $40 \%$ line may also suffer from the limited angular resolution effect mentioned above, but this is not a large problem for this data set. The limited resolution is clearly seen in the large scatter of the parallel to perpendicular temperature ratios, as discussed in Sect. 4.3, but it is possible to estimate both parallel and perpendicular 

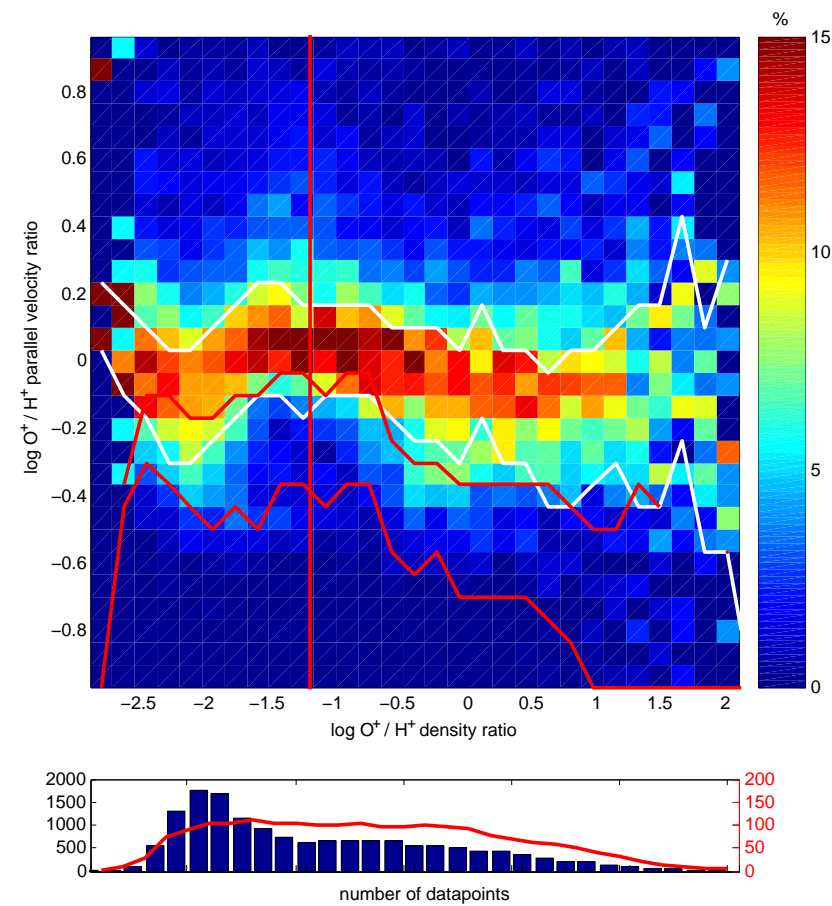

Fig. 4. Upper panel: Distribution of parallel bulk velocity ratio of $\mathrm{O}^{+}$over $\mathrm{H}^{+}$for each interval of $\mathrm{O}^{+}$over $\mathrm{H}^{+}$particle density ratio (log scale). Each column is normalized, the sum of all data bins in a column is $100 \%$. White lines indicate the narrowest region around the maximum in each column which contains $50 \%$ of all data points. The two similar red lines show the same distribution limits for the case when the $\mathrm{H}^{+}$moments were calculated for upward moving particles only (see text for further details). The vertical red line indicates where the mass density ratio of $\mathrm{H}^{+}$and $\mathrm{O}^{+}$is unity. Lower panel: number of data points contributing to each column (blue bars, left y-axis), and the number of measurement days contributing to the column (red line, right y-axis).

temperatures from the data. One may also note that for the higher bulk velocities (most pronounced above $100 \mathrm{~km} \mathrm{~s}^{-1}$ ) the thermal velocity typically exceeds the bulk velocity, i.e. the distribution has lost its beam character.

\section{Results}

The statistical results will all be shown in the same format used in Fig. 4 which we will take as an example to describe the format. The upper panel shows the occurrence frequency of the data on the y-axis for each corresponding data on the $\mathrm{x}$-axis. The data is normalized for each column. Thus if all data is contained in the plot, the sum of the occurrence frequency over each column is $100 \%$. In Fig. 4 the ratio of the field-aligned (parallel) component of the bulk drift velocity of $\mathrm{O}^{+}$and $\mathrm{H}^{+}$is compared with the density ratio of the two ion species. The density ratio is found on the $\mathrm{x}$-axis, and the plot thus illustrates the relative occurrence of different paral- lel velocity ratios for a given density ratio. Two white lines, one above and one below the peak occurrence frequency, indicate the narrowest region which contains $50 \%$ of the data points. Two similar red lines are also shown but these are specific for this particular plot and will be discussed later. The lower panel of the figure indicates the statistical significance of each column by showing the number of data-points contributing to each column (dark blue bars, left y-axis scale) and the number of different days contributing to each column (red line, right $y$-axis scale). The latter is important, because a large number of different days contributing to the same result indicates that what is seen is common. If almost every data point of a few days contribute to the same column the result will seem significant from the number of data points but the result need not be generally valid.

\subsection{Parallel bulk velocity relation}

To investigate question (1) posed in the introduction we compare the parallel bulk velocities of $\mathrm{H}^{+}$and $\mathrm{O}^{+}$in Fig. 4. This is done by plotting the $\mathrm{O}^{+}$to $\mathrm{H}^{+}$parallel velocity ratio as function of the $\mathrm{O}^{+}$to $\mathrm{H}^{+}$density ratio. The observations were made poleward of the cusp where the observed $\mathrm{H}^{+}$ fluxes are also flowing out, having mirrored in the Earth's magnetic field. Therefore the direction of flow is the same for almost all cases. The few cases of downgoing $\mathrm{H}^{+}$do not make a significant contribution and are not included in Fig. 4 as it is the logarithm of the velocity ratio which is shown.

It can immediately be seen in Fig. 4 that the distribution peaks where the parallel bulk velocities are close to the same ( 0 ratio on the log scale). The $50 \%$ lines are relatively close to the central peak and typically within \pm 0.2 on the log scale which means that the two parallel velocities are within a factor 1.5 of each other. Thus close to the same parallel bulk velocity for the two ion species is the most common situation, not just frequent enough to stand out in the statistical distribution. It is particularly noteworthy that the parallel bulk velocity ratio is close to unity for a wide range of density ratios. A vertical red line in Fig. 4 indicates the particle density ratio where the mass density ratio is unity. This shows that the $\mathrm{O}^{+}$population dominates in mass for a significant part of these cases. This may be important if a two-stream interaction is the explanation for the close to similar parallel velocity ratio.

In paper 1 better agreement was found when the $\mathrm{H}^{+}$ moment was calculated for upward moving particles only. Therefore we have investigated the same relation as was shown in Fig. 4 but with the $\mathrm{H}^{+}$moments calculated for upward moving particles only. The result is shown by including the smallest region which contains $50 \%$ of the data as two red lines in Fig. 4. As can be seen the distribution is now shifted towards higher $\mathrm{H}^{+}$than $\mathrm{O}^{+}$velocity. Clearly the parallel bulk velocity of the two ion species is in general much more similar when the full distribution is used for the 

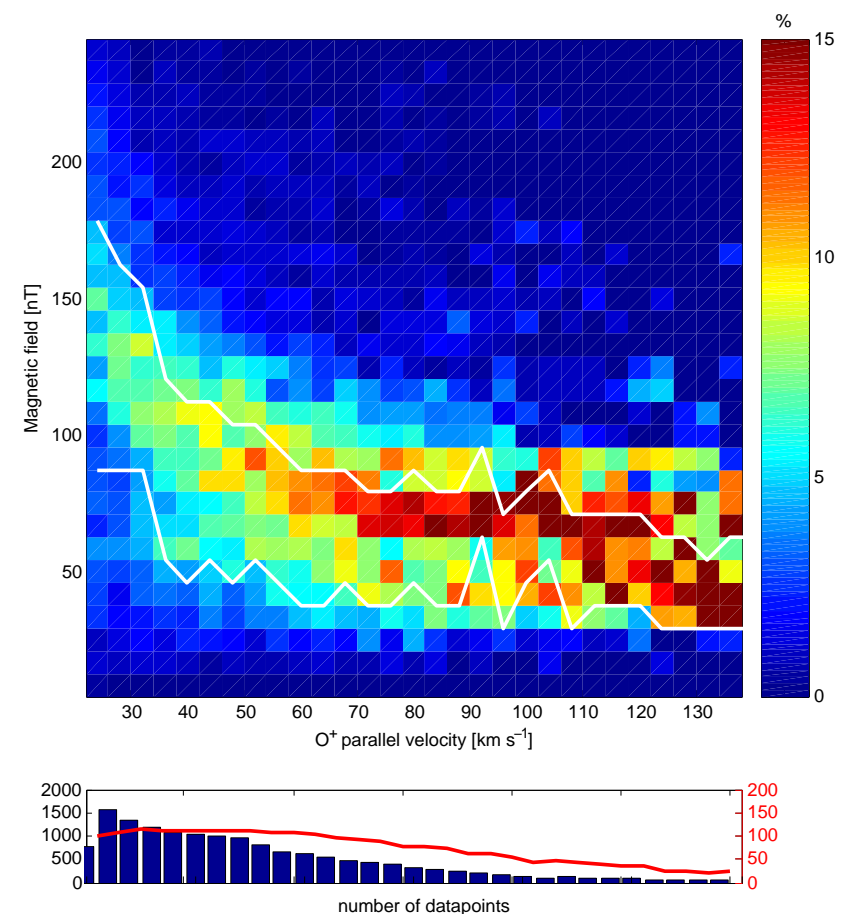

Fig. 5. Upper panel: Distribution of magnetic field intensity [nT] for each interval of $\mathrm{O}^{+}$parallel velocity $\left[\mathrm{km} \mathrm{s}^{-1}\right]$. Each column is normalized, the sum of all data bins in a column is $100 \%$. White lines indicate the narrowest region around the maximum in each column which contains $50 \%$ of all data points. Lower panel: Number of data points contributing to each column (blue bars, left y-scale), and the number of measurement days contributing to the column (red line, right y-scale).

$\mathrm{H}^{+}$moments. In the following section we will also compare the parallel bulk velocity ratio as a function of magnetic field strength / altitude.

\subsection{Altitude dependence}

To contribute to question (2) posed in the introduction we compare the parallel velocity and perpendicular temperature of $\mathrm{O}^{+}$to the magnetic field strength. The magnetic field is used as a proxy for altitude because of the importance of the magnetic mirror force. For passive (adiabatic) transport along the field-lines the mirror force will transfer perpendicular energy to parallel energy, and the perpendicular temperature should thus decrease with altitude.

Figure 5 shows the distribution of magnetic field intensity for each interval of parallel velocity. This is opposite to what is otherwise used in the paper where the distribution for each magnetic field intensity interval is shown. This is because a large range of high velocities can be seen for the lowest magnetic field intensities. This is now clearly seen in the plot, whereas the large spread of different relatively high magnetic field values for the low parallel velocity yields a
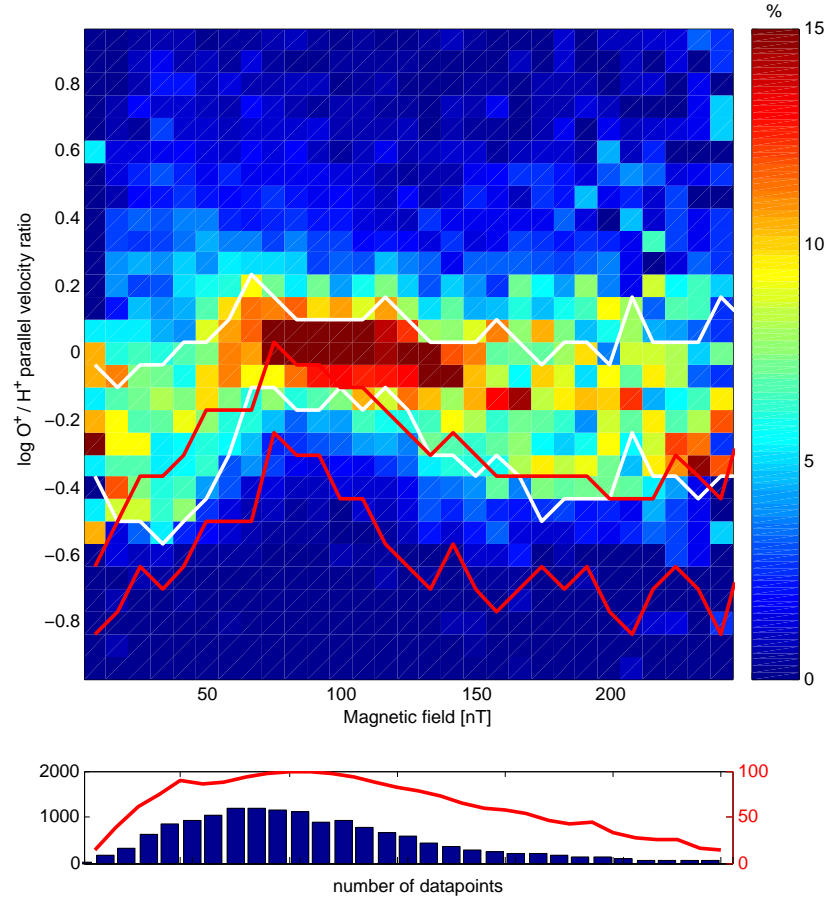

Fig. 6. Upper panel: Distribution of logarithm of $\mathrm{O}^{+}$to $\mathrm{H}^{+}$bulk parallel velocity ratio for each interval of magnetic field intensity [nT]. Each column is normalized, the sum of all data bins in a column is $100 \%$. White lines indicate the narrowest region around the maximum in each column which contains $50 \%$ of all data points. Similar red lines indicates the same but for $\mathrm{H}^{+}$moments calculated for upward moving particles only. Lower panel: number of data points contributing to each column (blue bars, left y-scale), and the number of measurement days contributing to the column (red line, right $\mathrm{y}$-scale).

rather indistinct peak in the distribution. Plotting the variables the other way around would yield the opposite visual result (though the meaning of the plot would be the same). It is clearly seen that the highest velocities are only seen at low magnetic field intensities, but there is otherwise no strong and simple relation between observed magnetic field intensity and parallel velocity of $\mathrm{O}^{+}$.

We also complement the discussion about the parallel bulk velocity ratio of $\mathrm{O}^{+}$and $\mathrm{H}^{+}$of the previous section by showing the altitude dependence of this ratio in Fig. 6. As can be seen the ratio is closest to unity in the altitude range corresponding to magnetic field-intensities between 50 and $150 \mathrm{nT}$ (corresponding on average to geocentric distances of 11.7 and 7.5 $R_{E}$ respectively for our data set). The velocity ratio is typically above -0.6 on the logarithmic scale throughout the observation interval which means that the $\mathrm{H}^{+}$bulk velocity typically is less than 4 times higher than $\mathrm{O}^{+}$. Thus the parallel bulk energy of $\mathrm{O}^{+}$is essentially always higher than that of $\mathrm{H}^{+}$. Two red lines in Fig. 6 indicate the narrowest region which contains $50 \%$ of the data when the $\mathrm{H}^{+}$moments are 

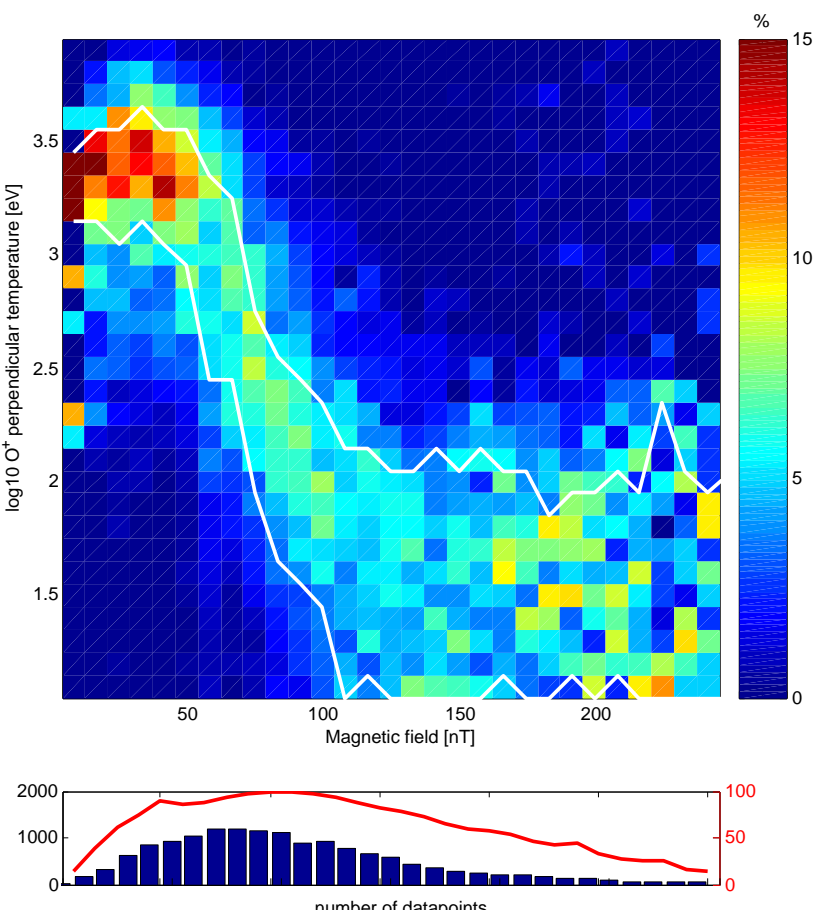

Fig. 7. Upper panel: Distribution of $\mathrm{O}^{+}$perpendicular temperature $[\log \mathrm{eV}]$ for each interval of magnetic field intensity [nT]. Each column is normalized, the sum of all data bins in a column is $100 \%$. White lines indicate the narrowest region around the maximum in each column which contains $50 \%$ of all data. Lower panel: number of data points contributing to each column (blue bars, left y-scale), and the number of measurement days contributing to the column (red line, right y-scale).

calculated for upward moving particles only. The $\mathrm{H}^{+}$bulk parallel velocity is then consistently higher for the $\mathrm{H}^{+}$than for $\mathrm{O}^{+}$, and agreement is much less close. The two velocities are closest at around $75 \mathrm{nT}$, and slope down towards a velocity ratio of about 4 (logarithm -0.6) on both sides of this maximum. Clearly the similarity between the two parallel bulk velocities is much higher for all altitude intervals when the $\mathrm{H}^{+}$moments are calculated for all particles, not just upward moving.

We now turn to the distribution of the perpendicular temperature with altitude (magnetic field strength). The perpendicular energy clearly increases for magnetic field values below $150 \mathrm{nT}$ as can be seen in Fig. 7. This increase with decreasing magnetic field / increasing altitude indicates high altitude perpendicular energization because for adiabatic transport we would expect the temperature to fall due to the action of the mirror force. The temperature increases from a few tens of $\mathrm{eV}$ to above $1 \mathrm{keV}$ at the highest altitudes (lowest magnetic field)). A similar plot for $\mathrm{H}^{+}$is shown in Fig. 8. The tendency is similar as for $\mathrm{O}^{+}$below $150 \mathrm{nT}$, but increases from just below $100 \mathrm{eV}$ up to about $500 \mathrm{eV}$. The $\mathrm{H}^{+}$temperature exhibits a clear minimum at magnetic field
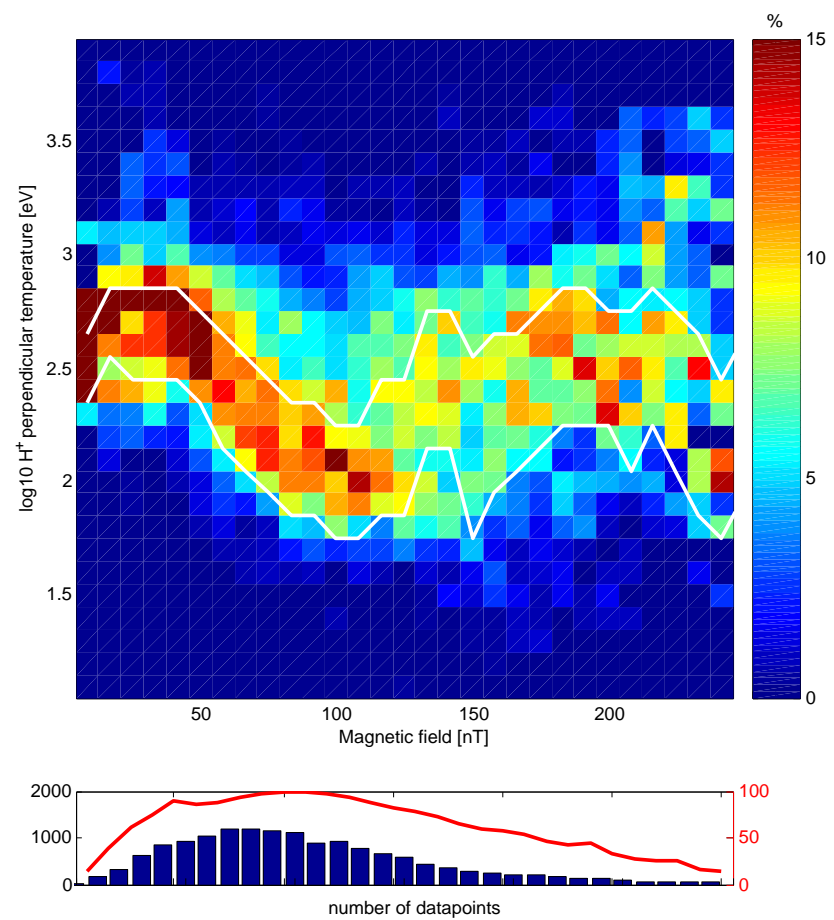

Fig. 8. Upper panel: distribution of $\mathrm{H}^{+}$perpendicular temperature [log eV] for each interval of magnetic field intensity [nT]. Each column is normalized, the sum of all data bins in a column is $100 \%$. White lines indicate the narrowest region around the maximum in each column which contains $50 \%$ of all data. Lower panel: number of data points contributing to each column (blue bars, left y-scale), and the number of measurement days contributing to the column (red line, right y-scale.

values of $100 \mathrm{nT}$. For higher values the temperature is approximately the same as for the lowest magnetic field values, a few hundred $\mathrm{eV}$. The assumed source of intense fluxes of $\mathrm{H}^{+}$is the magnetosheath, where the typical temperature is 100 to $200 \mathrm{eV}$. The most frequently observed temperature for $\mathrm{H}^{+}$at $100 \mathrm{nT}$ is thus at or below the lowest expected temperature of the magnetosheath source. The most frequently observed temperature at $50 \mathrm{nT}$ is more than twice the expected source temperature and a significant amount of samples are seen above $1 \mathrm{keV}$. Clearly the $\mathrm{H}^{+}$ions are also energized compared to the source in the magnetosheath, in the same region as where the $\mathrm{O}^{+}$ions are energized. However, as the main $\mathrm{H}^{+}$source is above the mirror field of the Earth's magnetosphere, we cannot tell if they were energized on their way up or down. Significant energization of downgoing magnetosheath $\mathrm{H}^{+}$fluxes would lead to mirroring at higher altitudes than without the heating and contribute to a net outflow of $\mathrm{H}^{+}$. In both cases the heating would contribute to subsequent outflow of both ion species, irrespective of the initial direction of flow of $\mathrm{H}^{+}$.

In the previous section we compared two simultaneously observed moments with each other which is a very robust 

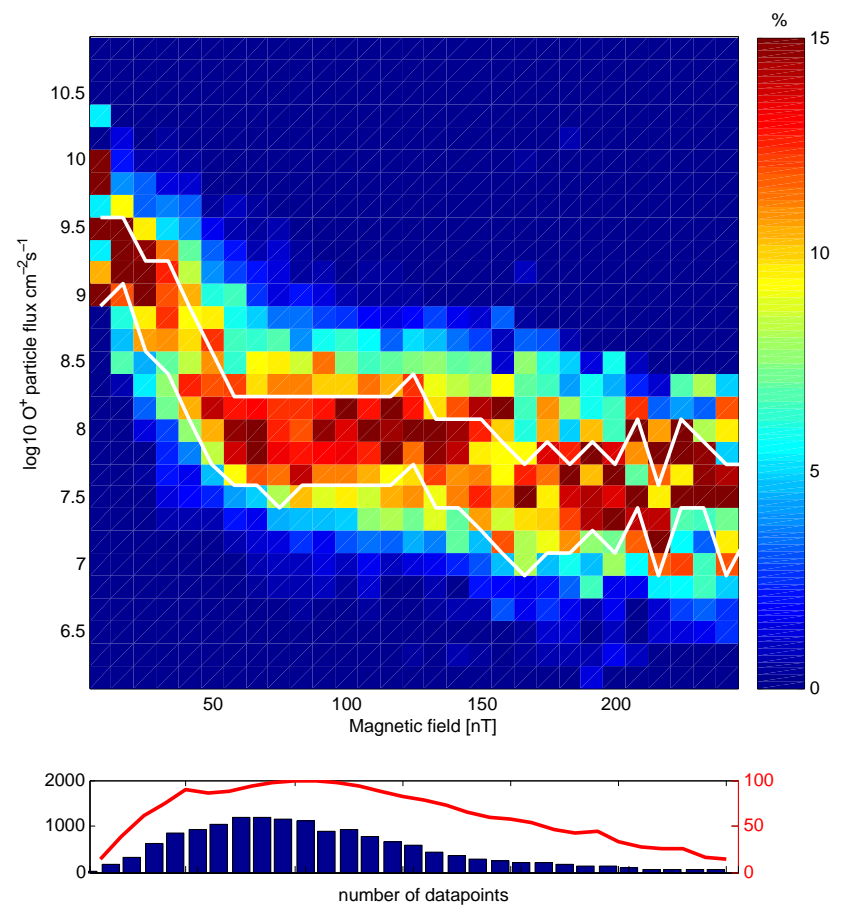

Fig. 9. Upper panel: distribution of $\mathrm{O}^{+}$particle number flux $\left[\log \mathrm{cm}^{-2} \mathrm{~s}^{-1}\right]$ for each interval of magnetic field intensity [nT]. Each column is normalized, the sum of all data bins in a column is $100 \%$. White lines indicate the narrowest region around the maximum in each column which contains $50 \%$ of all data. Lower panel: number of data points contributing to each column (blue bars, left $y$-scale), and the number of measurement days contributing to the column (red line, right y-scale.

comparison with few underlying assumptions. For the comparison with magnetic field and (implied) altitude the situation is a bit more complicated. For each individual orbit of the satellite the bulk drift energy and temperature decrease at higher latitudes (and for a typical orbit this also means lower altitude and higher magnetic field) which may be due to a velocity dispersion effect. Far away from the source (the open magnetopause of the cusp for magnetosheath origin $\mathrm{H}^{+}$) only the slowest particles from the initial injection still remain. The apparent altitude dependence can thus be a latitude dependence. For our statistical data set the orbits vary and sample the same latitude at different altitudes, but effects due to a biased sampling in latitude-altitude space may still remain. Comparing the data versus estimated invariant latitude of the satellite instead of magnetic field value yields a much weaker or no correlation judging from a plot of the same type as in Figs. 7 and 8. This can be quantified by calculating the cross-correlation coefficients between the data sets. These are for the relation logarithm of $\mathrm{O}^{+}$temperature to magnetic field strength and invariant latitude -0.57 and -0.011 respectively, for magnetic field strengths between 50 and $150 \mathrm{nT}$. For $\mathrm{H}^{+}$these coefficients are -0.17 and -0.048 for magnetic
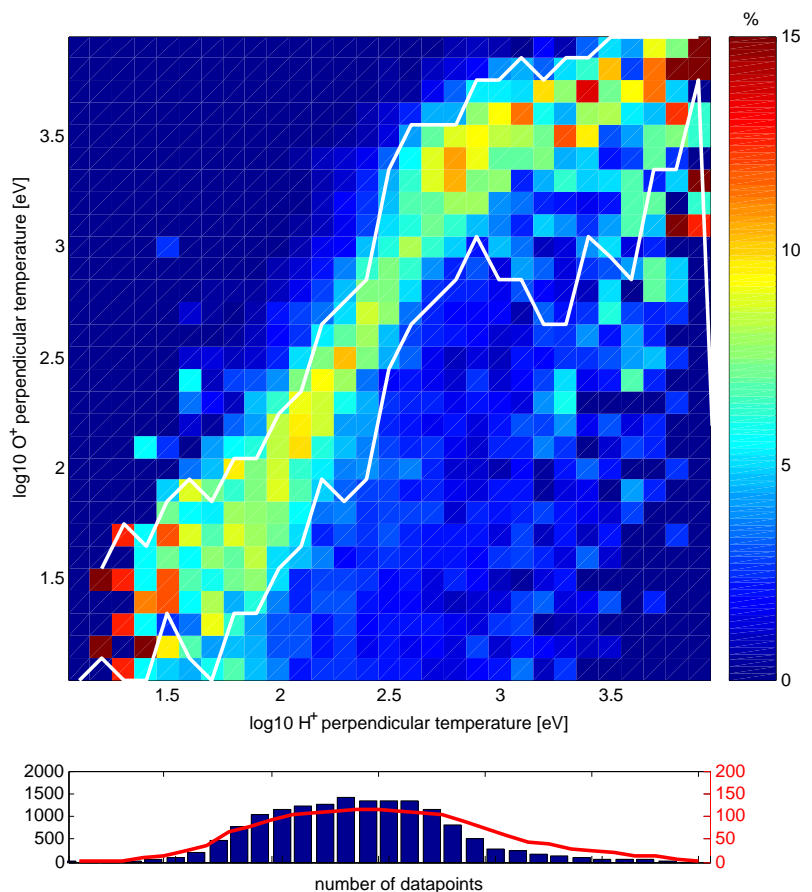

Fig. 10. Upper panel: distribution of $\mathrm{O}^{+}$perpendicular temperature [log eV] for each interval of $\mathrm{H}^{+}$perpendicular temperature [log $\mathrm{eV}$. Each column is normalized, the sum of all data bins in a column is $100 \%$. White lines indicate the narrowest region around the maximum in each column which contains $50 \%$ of all data. Lower panel: number of data points contributing to each column (blue bars, left y-scale), and the number of measurement days contributing to the column (red line, right y-scale).

field strengths between 50 and $100 \mathrm{nT}$. Finally one may check the correlation coefficient for magnetic field strength and invariant latitude for our data set which is 0.0244 for magnetic fields between 50 and $150 \mathrm{nT}$. Thus there is some bias in the latitude-altitude interval, but it is much weaker than the correlations found between magnetic field (altitude) and perpendicular temperature. A final check can be made by looking at the particle number flux for $\mathrm{O}^{+}$, normalized to a magnetic field of $50000 \mathrm{nT}$ (ionospheric altitude). The normalization is made to take into account the widening of the flux tube. If no new particles are introduced at higher altitudes there should not be an altitude dependence of the particle flux. In principle cold particles could be brought into the instrument measurement range at any altitude but significant counts are only seen in the lowest energy channels at low altitude when the entire $\mathrm{O}^{+}$beam has a low energy (see Fig. 1). A plot of $\mathrm{O}^{+}$particle flux vs. magnetic field strength is shown in Fig. 9.

As can be seen the particle flux is fairly constant for magnetic field values between 50 and $150 \mathrm{nT}$. The generally lower fluxes at higher magnetic field values and the higher fluxes at low magnetic field values are likely latitude effects. 

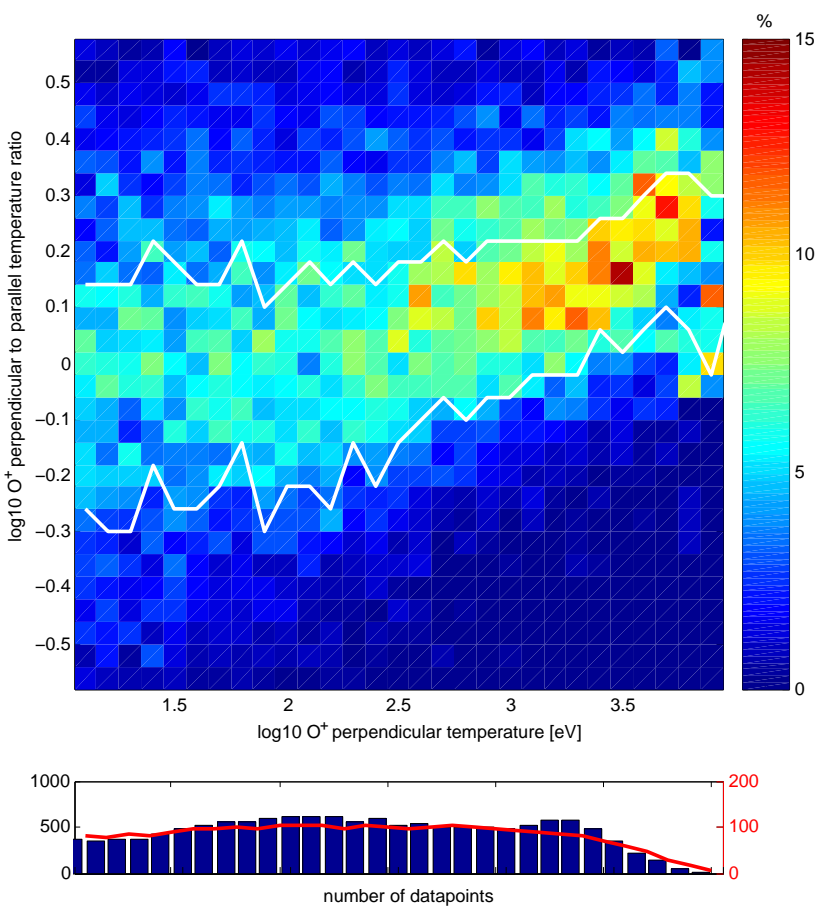

Fig. 11. Upper panel: distribution of logarithm of $\mathrm{O}^{+}$perpendicular to parallel temperature ratio for each interval of $\mathrm{O}^{+}$perpendicular temperature $[\log \mathrm{eV}]$. Each column is normalized, the sum of all data bins in a column is $100 \%$. White lines indicate the narrowest region around the maximum in each column which contains $50 \%$ of all data. Lower panel: number of data points contributing to each column (blue bars, left y-scale), and the number of measurement days contributing to the column (red line, right y-scale).

To study latitude dependence in detail one must compare with the distance to the cusp/injection region and take the observed convection into account, just a fixed invariant latitude is not good enough (e.g. Valek et al., 2002; Dubouloz et al., 2001). This will be left for a future study.

Finally it is worthwhile to also directly compare the perpendicular temperature of $\mathrm{O}^{+}$with that of $\mathrm{H}^{+}$, as shown in Fig. 10.

The relation is very tight, but it is not the linear relationship seen for the parallel bulk velocity. Instead the $\mathrm{O}^{+}$temperatures cover a much wider temperature span. The relation is $\log _{10} \mathrm{~T}_{\mathrm{O}^{+}}=1.55 \cdot \log _{10} \mathrm{~T}_{\mathrm{H}^{+}}-1.1$ for $\mathrm{H}^{+}$temperatures up to $1 \mathrm{keV}$. If both species were heated to the same thermal velocity, i.e. $\mathrm{O}^{+} 16$ times higher temperature than $\mathrm{H}^{+}$then the slope would still be 1 but with an offset of 1.2 on the logarithmic scale. The difference between $\mathrm{O}^{+}$and $\mathrm{H}^{+}$temperature never reaches 1.2 on the log scale in Fig. 10. Therefore even if the parallel velocity ratio is close to one-to-one throughout the observation region the thermal velocity is never the same for the two ion species and the temperature ratio for the two species changes with altitude. For the highest temperatures the difference in Fig. 10 is about 0.5 on the log scale corresponding to a factor 3 difference. The high-energy cutoff of the instrument may to some extent affect this result, but very few datapoints show temperatures above $10 \mathrm{keV}$.

\subsection{Perpendicular to parallel temperature ratios}

In order to contribute to question (3) posed in the introduction we show in Fig. 11 the distribution of the logarithm of perpendicular to parallel temperature ratio of $\mathrm{O}^{+}$for a given perpendicular $\mathrm{O}^{+}$temperature.

For temperatures below a few hundred $\mathrm{eV}$ there is large scatter around 0 (i.e. isotropic distribution). This is consistent with the temperature typically being smaller than the bulk kinetic drift energy for these temperatures (Fig. 3) thus making accurate temperature and in particular temperature anisotropy estimates more difficult. Above $1 \mathrm{keV}$ temperature the data is much less scattered and shows a clear tendency towards higher perpendicular than parallel temperatures. This is often a signature of local heating, as the mirror force will decrease the kinetic perpendicular temperature if the ions move up along the field-line. Ions may in principle have been energized in both perpendicular and downward direction above the observation point, but once again the mirror force will limit how far such a population can move down the field-line before it mirrors.

\section{Discussion}

\subsection{Nearly equal $\mathrm{H}^{+}$and $\mathrm{O}^{+}$parallel velocities}

Figures 4 and 6 show very clearly that nearly equal fieldaligned velocity is the most common situation for $\mathrm{H}^{+}$and $\mathrm{O}^{+}$observed in the polar cap / mantle region. There are essentially three ways to obtain this situation:

1. Both species can in some sense be said to come from the same limited source region and particles with the same velocity are seen at the same time some distance away from the source (a time-of-flight effect).

2. Both ion species are energized to nearly the same velocity.

3. Energy is transferred between the populations through a two-stream interaction.

Possibility (1) cannot be the explanation for our observations, because the $\mathrm{H}^{+}$population is warm (high temperature compared to bulk drift velocity), and a time-of-flight effect would make only a narrow range of velocities (energies) be seen at a significant distance from the source (see paper 1 for further discussion on the velocity dispersion effect). This also means that it is not so simple that once two populations are moving with the same velocity they will stay together. The $\mathrm{H}^{+}$has a high thermal velocity compared to the bulk 
drift velocity. Only a narrow part of the population will actually follow the simultaneously observed (colder) $\mathrm{O}^{+}$population. Note that for the polar cap it is possible that a region limited in both altitude and latitude associated with the cusp is a feasible source for both magnetosheath origin ions and energized $\mathrm{O}^{+}$ions observed over the polar cap (e.g. Bouhram et al., 2003; Bogdanova et al., 2004). Magnetosheath ions enter the magnetosphere and mirror in this region and $\mathrm{O}^{+}$ions get energized to significant energies in the same region. As discussed in paper 1 much of the narrowness in energy of the $\mathrm{O}^{+}$beam may be due to velocity dispersion of ions emanating from such a source region. However this cannot explain the similarity between the parallel bulk velocity of the two species as the velocity distributions are so different.

Possibility (2) may explain the observations of similar $\mathrm{H}^{+}$ and $\mathrm{O}^{+}$parallel velocities if it can be said that the initially more energetic $\mathrm{H}^{+}$ions are also further energized. If the energization mechanism is more efficient for cold and heavy particles the initially cold heavier particles may quickly catch up. Energization of particles of different mass up to the same velocity rather than the same energy is discussed in some publications, e.g. acceleration associated with drifting inhomogeneities (Antonova, 1983), through the ponderomotive force (e.g. Guglielmi and Lundin, 2001, and references therein), through stochastic acceleration in Alfvén waves (e.g. Chaston et al., 2004, and references therein) and finally field-aligned acceleration associated with the convection electric field and curved magnetic field lines, termed centrifugal acceleration (Cladis, 1986; Cladis et al., 2000). We will first consider the mechanisms which achieve parallel acceleration through initial perpendicular heating. There is indication in the perpendicular ion temperature data that the $\mathrm{H}^{+}$population is energized in the perpendicular direction as the reflected magnetosheath particles travel out along the field-lines (see Fig. 8 and Sect. 4.2). The details of this is discussed in Sect. 5.2. Even though our data confirm a stronger heating for $\mathrm{O}^{+}$than for $\mathrm{H}^{+}$we never see the same thermal velocity for the two ion species, whereas the similarity in parallel bulk velocity is seen throughout the observation region. Therefore perpendicular heating contributes, giving $\mathrm{O}^{+}$a higher energy than $\mathrm{H}^{+}$, but not enough to reach the same parallel velocity. Furthermore perpendicular heating to the same velocity and subsequent outflow involves only upward moving particles and cannot explain why the similarity is better when the bulk velocity of $\mathrm{H}^{+}$is calculated for all particles.

The so called centrifugal force mechanism on the other hand may provide precisely the needed further parallel acceleration. Whereas it cannot explain the perpendicular heating of $\mathrm{O}^{+}$it contributes to the increase of parallel velocity. Acceleration occurs when the magnetic field change of direction has a component along the $\boldsymbol{E} \times \boldsymbol{B}$ drift velocity (Cladis, 1986; Northrop, 1963; Horwitz et al., 1994). The velocity increase depends on variables used in this study: the perpendicular drift velocity and magnetic field direction, and when the change of magnetic field-direction occurs as the particles move along the field-line it also depends on the field-aligned velocity of the particles. The latter also determines for how long time the particle experiences the acceleration so that the parallel velocity will not matter for the finally achieved velocity increase. Making a proper estimate of the centrifugal acceleration requires estimates of the field-line change of direction along the field-line, along the convection direction and with time. This is difficult to do properly but a multispacecraft technique could lead to a good estimate. Using a dipole magnetic field and the observed convection for our data-set we obtain a typical velocity increase due to changing magnetic field-direction along the magnetic field-line of a few $\mathrm{km} \mathrm{s}^{-1}$ to a few $10 \mathrm{~km} \mathrm{~s}^{-1}$ per Earth radii $\left(R_{E}\right)$ travel distance along time-independent field-lines. This is in agreement with the calculations of Cladis (1986) who used a Kp dependent magnetospheric magnetic field model and a model convection electric field. In the model of Cladis (1986) the particles reached about $100 \mathrm{eV}$ energy $\left(35 \mathrm{~km} \mathrm{~s}^{-1}\right)$ after $2 \mathrm{~h}$ travel time along the highest altitude orbits (their trajectory 1) which should be most similar to our high-altitude trajectories. Energies above $1 \mathrm{keV}$ (above $100 \mathrm{~km} \mathrm{~s}^{-1}$ ) were mainly found in the central plasma sheet region where the field-line radius of curvature is small. This still indicates that the centrifugal acceleration is a feasible parallel acceleration mechanism which can partially explain the high and similar parallel velocities of $\mathrm{H}^{+}$and $\mathrm{O}^{+}$above the polar cap. Centrifugal acceleration cannot explain why the similarity is most pronounced when the full distribution function of $\mathrm{H}^{+}$is used.

Possibility (3) can clearly explain the relation between the parallel bulk velocity of $\mathrm{H}^{+}$and $\mathrm{O}^{+}$reported in this paper. According to the work of Ludlow and Kaufman (1989) a two-stream interaction may also lead to some perpendicular heating of the $\mathrm{O}^{+}$population. In paper 1 the $\mathrm{O}^{+}$parallel velocity was even higher than that for $\mathrm{H}^{+}$, which seems inconsistent with a two-stream interaction. However when the $\mathrm{H}^{+}$velocity was calculated for only upward moving particles the velocities were close to the same. We can note that in the statistical data presented in this paper this is not the typical situation. Using $\mathrm{H}^{+}$moments calculated only for the upward moving population does not improve the statistics at all. What speaks strongly for a two-stream interaction is that this very obviously should lead to close to the same parallel velocity for the two ion species and it is plausible that also downward moving $\mathrm{H}^{+}$particles can have an influence. The other mechanisms can lead to a similar velocity increase, but when considering the different source locations (ionosphere vs. magnetosheath) and source temperatures (of the order of $1 \mathrm{eV}$ vs $100 \mathrm{eV}$ ) it is not obvious that close to the same parallel velocity should be the final result. What speaks against the twostream interaction is that the parallel velocity is close to the same for a large range of relative abundance of the two particle species. There is not one faster major species from which energy is transferred to the same minor species for all conditions. 


\subsection{High altitude heating of $\mathrm{H}^{+}$and $\mathrm{O}^{+}$}

It has already been shown by Arvelius et al. (2005) that additional energization occur for $\mathrm{O}^{+}$at high altitudes above the polar cap. Our observation of increasing perpendicular temperature for high altitude (low magnetic field) shows that there is persistent perpendicular heating of ionospheric origin ions over extended altitude intervals up to the highest observed distances (about $12 R_{E}$ ). The perpendicular to parallel temperature ratio further emphasize high altitude heating by showing a preference for higher perpendicular to parallel temperature ratios for the highest observed temperatures (which occur at low magnetic field / high altitude). There exists a clear relation between observed $\mathrm{H}^{+}$and $\mathrm{O}^{+}$perpendicular temperature (Fig. 10). The strong increase of $\mathrm{O}^{+}$ temperature with altitude, despite the action of the mirror force, as well as the tight coupling between the temperature of the two species points towards a mechanism which preferentially heats $\mathrm{O}^{+}$. This need not be one of the mechanisms mentioned under possibility (2) in the previous section, in principle a wave frequency spectrum which is stronger at the $\mathrm{O}^{+}$gyro frequency than at the $\mathrm{H}^{+}$gyro frequency could lead to stronger heating of $\mathrm{O}^{+}$. However the strong coupling in temperature apparent in Fig. 10 should in such a case result from the altitude dependence of the temperature of the two ion species. However the relation between the temperature of the two species is much tighter than the altitude dependence of the $\mathrm{H}^{+}$temperature (compare Figs. 8 and 10).

One may finally note that whereas the relation between perpendicular ion temperature and magnetic field, as well as between parallel velocities and perpendicular temperatures of the two ion species, are strong, the relation between parallel velocity and magnetic field is not so strong. Clearly the highest velocities (above $60 \mathrm{~km} \mathrm{~s}^{-1}$ are only observed at the lowest magnetic field values (below $100 \mathrm{nT}$ ), but within that region no clear relation can be seen between the two parameters. This seems to indicate a two stage process: (1) heating which is more effective for heavy ions and (2) a coupling between the parallel bulk velocities of the two ion species, either through a two-stream interaction or a species dependent field-aligned energization such as the centrifugal force. The bulk parallel velocity of $\mathrm{O}^{+}$seems to be an effect of these two processes acting together rather than there being some single clear species dependent parallel energization mechanism.

\subsection{Perpendicular to parallel temperature ratio of $\mathrm{O}^{+}$}

In paper 1 it was noted that the $\mathrm{O}^{+}$temperature had a tendency towards isotropy and that this could be explained by heating over extended altitude intervals in combination with adiabatic transport (i.e. the influence of the mirror force). In paper 3 this was investigated further and this result was confirmed. Furthermore paper 3 showed that there were instances of strong heating that appeared limited in space and time. Our statistical study confirms that higher perpendicular than parallel thermal velocity is often observed at the highest altitudes. It is therefore worthwhile to use the statistical database to find clear cases of high perpendicular to parallel temperature ratios and investigate the wave data for these events. This way it may be possible to learn more about the details of the heating mechanism. Relating the observations to wave data is more difficult than characterizing the particle data as we have done in this paper. This is because the actual heating and/or two-stream interaction need not take place just when/where the observations were made (if it is sporadic and or limited in time as suggested by the results presented in paper 3). However it must reasonably take place on some occasions when and where the observations were made and the statistical data base is suitable to chose some likely cases, i.e. when the perpendicular to parallel temperature ratio or the $\mathrm{H}^{+}$to $\mathrm{O}^{+}$parallel bulk velocity ratio was high. This will be done in a future study.

\section{Conclusions}

We have made a statistical characterization of high altitude $\left(6-12 R_{E}\right) \mathrm{O}^{+}$beams and the typical relation to simultaneously observed $\mathrm{H}^{+}$fluxes of magnetosheath origin. It was found that the temperature of $\mathrm{O}^{+}$increases strongly with altitude and the associated decreasing magnetic field value despite the effect of the mirror force. The $\mathrm{O}^{+}$perpendicular temperature typically increases from a few tens of $\mathrm{eV}$ at $150 \mathrm{nT}$ magnetic field strength to a few $\mathrm{keV}$ at $50 \mathrm{nT}$. For the highest temperatures observed there is a tendency towards an anisotropic temperature distribution with higher perpendicular than parallel temperature. Furthermore the perpendicular temperatures of $\mathrm{H}^{+}$and $\mathrm{O}^{+}$appear coupled. There is not a linear relation, rather the increase is much steeper for $\mathrm{O}^{+}$, with a factor of about 1.5 in the logarithmic relation.

Another very clear relation between $\mathrm{O}^{+}$and $\mathrm{H}^{+}$is that the parallel bulk velocity is typically close to the same. Heating of both ion species up to the same velocity can explain both the coupling between temperatures and parallel bulk velocities. However the thermal velocity of $\mathrm{O}^{+}$is always below that of $\mathrm{H}^{+}$whereas the bulk velocity is close to the same throughout the observation region. At the highest observed temperatures (and thus altitudes) there is a factor of about 3 difference between the temperatures of the ion species, whereas a factor 16 is needed for the same thermal velocity. Thus in addition to the perpendicular heating of $\mathrm{O}^{+}$ there must also be a parallel acceleration mechanism which provides a similar parallel velocity to the two ion species. This can either be a two-stream interaction or the so called centrifugal acceleration caused by the curvature of the magnetic field-lines in the direction of the magnetospheric convection. A rough estimate indicates that the latter is significant for our cases, yielding velocity increases of from a few to a few $10 \mathrm{~km} \mathrm{~s}^{-1}$ per $R_{E}$ travel along the field-line. This 
is approximately enough to explain the observations, but a more advanced analysis of the centrifugal acceleration must be performed before a more final statement can be made.

The parallel bulk velocity of the two ion species is close to the same for a large range of relative abundance of the two ion species. This may be a problem for an explanation involving two-stream interaction, and certainly must be taken into account when such an explanation is investigated in detail. Speaking for some kind of interaction between the two ion populations is the fact that the parallel bulk velocity is most similar when the $\mathrm{H}^{+}$bulk velocity is calculated for all particles, not just upward moving. The other mechanisms essentially concern similar acceleration of outward moving particles and the downgoing part of the $\mathrm{H}^{+}$population should not play a role.

This statistical study needs to be followed up with cases studies investigating occasions when heating and the twostream interaction seems likely to be taking place at the observation points (i.e. high perpendicular to parallel temperature ratio and high $\mathrm{H}^{+}$to $\mathrm{O}^{+}$parallel bulk velocity ratio). Furthermore it should be possible to make a rather precise estimate of the centrifugal acceleration of both mirroring magnetosheath ions and escaping ionospheric origin oxygen ions using test particles in a model magnetic field and a multispacecraft data analysis technique to separate observed fieldline direction changes into field-aligned, field-perpendicular and temporal components.

Acknowledgements. This work was supported by the National Space Board of Sweden.

Topical Editor I. A. Daglis thanks J. Slavin and O. W. Lennartsson for their help in evaluating this paper.

\section{References}

Andersson, L., Peterson, W. K., and McBryde, K. M.: Estimates of the suprathermal $\mathrm{O}^{+}$outflow characteristic energy and relative location in the auroral oval, Geophys. Res. Lett., 32, doi:10.1029/2004GL021434, 2005.

André, M. and Yau, A. W.: Theories and observations of ion energization and outflow in the high latitude magnetosphere, Space Sci. Rev., 80, 27-48, 1997.

Antonova, Y. Y.: Transverse acceleration of ionospheric ions induced by collisions with moving inhomogeneities, Geomag. Aeronomy, 23, 610-612, 1983.

Arvelius, S., Yamauchi, M., Nilsson, H., Lundin, R., Hobara, Y., Rème, H., Bavassano-Cattaneo, M. B., Paschmann, G., Korth, A., Kistler, L., and Parks, G. K.: Statistics of high-altitude and high-latitude $\mathrm{O}^{+}$ion outflows observed by Cluster/CIS, Ann. Geophys., 23, 1909-1916, 2005.

Balogh, A., Carr, C. M., Acuña, M. H., Dunlop, M. W., Beek, T. J., Brown, P., Fornaon, K.-H., Georgescu, E., Glassmeier, K.-H., Harris, J., Musmann, G., Oddy, T., and Schwingenschuh, K.: The Cluster Magnetic Field Investigation: overview of in-flight performance and initial results, Ann. Geophys., 19, 1207-1217, 2001.
Bergmann, R., Roth, I., and Hudson, M. K.: Linear stability of the $\mathrm{H}^{+}-\mathrm{O}^{+}$two-stream interaction in a magnetised plasma, J. Geophys. Res., 93, 4005-4020, 1988.

Bogdanova, Y. V., Fazakarley, A. N., Owen, C. J., Klecker, B., Cornilleau-Wehrlin, N., Grison, B., Andr' e, M., Cargill, P., Rème, H., Bosqued, J. M., Kistler, L. M., and Balogh, A.: Correlation between suprathermal electron bursts, broadband extremely low frequency waves, and local ion heating in the midaltitude cleft/low-altitude boundary layer observed by Cluster, J. Geophys. Res., 109, doi.10.1029/2004JA010554, 2004.

Bouhram, M., Malingre, M., Jasperse, J. R., and Dubouloz, N.: Modelling transverse heating and outflow of ionospheric ions from the dayside cusp/cleft. 1 A parametric study, Ann. Geophys., 21, 1753-1771, 2003.

Bouhram, M., Klecker, B., Miyake, W., Rème, H., Sauvaud, J.-A., Malingre, M., Kistler, L., and Blăgău, A.: On the altitude dependence of transversely heated $\mathrm{O}^{+}$distributions in the cusp/cleft, Ann. Geophys., 22, 1787-1798, 2004.

Chaston, C. C., Bonnell, J. W., Carlson, C. W., McFadden, J. P., Ergun, R. E., Strangeway, R. J., and Lund, E. J.: Auroral ion acceleration in dispersive Alfvén waves, J. Geophys. Res., 109, doi:10.1029/2003JA010053, 2004.

Cladis, J. B.: Parallel acceleration and transport of ions from polar ionosphere to plasmasheet, J. Geophys. Res., 13, 893-896, 1986.

Cladis, J. B., Collin, H. L., Lennartsson, O. W., Moore, T. E., Peterson, W. K., and Russell, C. T.: Observations of centrifugal acceleration during compression of magnetosphere, Geophys. Res. Lett., 27, 915-918, 2000.

Dubouloz, N., Bouhram, M., Senior, C., Delcourt, D., Malingre, M., and Sauvaud, J.-A.: Spatial structure of the cusp/cleft ion fountain: A case study using a magnetic conjugacy between Interball AP and a pair of SuperDARN radars, J. Geophys. Res., 106, 261-274, 2001.

Frank, L. A., Ackerson, K. L., and Yeager, D. M.: Observations of atomic oxygen $\left(\mathrm{O}^{+}\right)$in the Earth's magnetotail, J. Geophys. Res., 82, 129-133, 1977.

Guglielmi, A. and Lundin, R.: Ponderomotive upward acceleration of ions by ion cyclotron and Alfvén waves over the polar regions, J. Geophys. Res., 106, 13 219-13 236, 2001.

Horwitz, J. L., Ho, C. W., Scarbro, H. D., Wilson, G. R., and Moore, T. E.: Centrifugal acceleration of the polar wind, J. Geophys. Res., 99, 15 051-15 064, 1994.

Kistler, L. M., Frey, H. U., Møbius, E., Mouikis, C., Quinn, J. M., Klecker, B., Rème, H., Bosqued, J. M., Dandouras, I., Sauvaud, J. A., Di Lellis, A. M., Formisano, V., Marcucci, M. F., Carlson, C. W., McFadden, J. P., Parks, G. K., McCarthy, M., Korth, A. Eliasson, L., Lundin, R., Paschmann, G., Popecki, M. A., Mende, S. B., Winningham, J. D., and Fazakarley, A. N.: Motion of auroral ion outflow structures observed with CLUSTER and IMAGE FUV, J. Geophys. Res., 107, doi:10.1029/2001JA005075, 2002.

Klumpar, D., Peterson, W. K., and Shelley, E. G.: Direct evidence of for two-stage (bimodal) acceleration of ionospheric ions, J. Geophys. Res., 10779-10 787, 1984.

Krauklis, I., Johnstone, A. D., and Peterson, W. K.: Acceleration of ionospheric $\mathrm{O}^{+}$ions on open field lines in the Low-Latitude Boundary Layer and the cusp region, J. Geophys. Res., 106, 29611-29618, 2001. 
Lennartsson, O. W., Collin, H. L., and Peterson, W. K.: Solar wind control of Earth's $\mathrm{H}^{+}$and $\mathrm{O}^{+}$outflow rates in the $15-\mathrm{eV}$ to $33-\mathrm{keV}$ energy range, J. Geophys. Res, 109, doi:10.1029/2004JA010,690, 2004.

Ludlow, G. R. and Kaufman, R. L.: Heating of upflowing auroral $\mathrm{H}^{+}$and $\mathrm{O}^{+}$beams: results from quasi-linear theory, J. Geophys. Res., 94, 319-328, 1989.

Lundin, R., Zakharov, A., Pellinen, R., Hultqvist, B., Borg, H., Dubinin, E., Barabash, S., Pissarenko, N., Koskinen, H., and Liede, I.: First results of the ionospheric plasma escape from Mars, Nature, 341, 609-612, 1989.

Möbius, E., Tang, L., Kistler, L. M., Popecki, M., Lund, E. J., Klumpar, D., Peterson, W., Shelley, E., Klecker, B., Hovestadt, D., Carlson, C. W., Ergun, R., McFadden, J. P., Mozer, F., Temerin, M., Cattell, C., Elphic, R., Strangeway, R., and Pfaff, R.: Species dependent energies in upward directed ion beams over auroral arcs as observed with FAST TEAMS, Geophys. Res. Lett., 25, 2029-2032, 1998.

Moore, T. E., Lundin, R., Alcayde, D., André, M., Ganguli, S. B., Temerin, M., and Yau, A.: Source processes in the high-altitude ionosphere, Space Sci. Rev., 88, 7-84, 1999.

Nilsson, H., Yamauchi, M., Eliasson, L., Norberg, O., and Clemmons, J.: The ionospheric signature of the cusp as seen by incoherent scatter radar, J. Geophys. Res., 101, 10 947-10 963, 1996.

Nilsson, H., Joko, S., Lundin, R., R' eme, H., Sauvaud, J.-A., Dandouras, I., Balogh, A., Carr, C., Kistler, L. M., Klecker, B., Carlson, C. W., Bavassano-Cattaneo, M. B., and Korth, A.: The structure of high altitude $\mathrm{O}^{+}$energization and outflow: a case study, Ann. Geophys., 22, 2497-2506, 2004.

Norqvist, P., André, M., and Tryland, M.: A statistical study of ion energization mechanisms in the auroral region, J. Geophys. Res., 103, 23 459-23 474, 1998.

Northrop, T. G.: The adiabatic motion of charged particles, Interscience Publishers, N.Y., 1963.

Ogawa, Y., Fujii, R., Buchert, S. C., Nozawa, S., and Ohtani, S.: Simultaneous EISCAT Svalbard radar and DMSP observations of ion upflow in the dayside polar ionosphere, J. Geophys. Res., 108, doi:10.1029/2002JA009590, 2003.

Paschmann, G. and Daly, P., E.: Analysis methods for multi spacecraft measurements, Kluwer Academic Publishers, 1998.

Rème, H., Aoustin, C., Bosqued, J. M., Dandouras, I., Lavraud, B., Sauvaud, J. A., Barthe, A., Bouyssou, J., Camus, T., Coeur-Joly, O., Cros, A., Cuvilo, J., Ducay, F., Garbarowitz, Y., Medale, J. L., Penou, E., Perrier, H., Romefort, D., Rouzaud, J., Vallat, C., AlcaydE, D., Jacquey, C., Mazelle, C., d'Uston, C., Möbius, E., Kistler, L. M., Crocker, K., Granoff, M., Mouikis, C., Popecki, M., Vosbury, M., Klecker, B., Hovestadt, D., Kucharek, H., Kuenneth, E., Paschmann, G., Scholer, M., Sckopke, N., Seidenschwang, E., Carlson, C. W., Curtis, D. W., Ingraham, C., Lin, R. P., McFadden, J. P., Parks, G. K., Phan, T., Formisano, V., Amata, E., Bavassano-Cattaneo, M. B., Baldetti, P., Bruno, R., Chionchio, G., Lellis, A. D., Marcucci, M. F., Pallocchia, G., Korth, A., Daly, P. W., Graeve, B., Rosenbauer, H., Vasyliunas, V., McCarthy, M., Wilber, M., Eliasson, L., Lundin, R., Olsen, S., Shelley, E. G., Fuselier, S., Ghielmetti, A. G., Lennartsson, W., Escoubet, C. P., Balsiger, H., Friedel, R., Cao, J.-B., Kovrazhkin, R. A., Papamastorakis, I., Pellat, R., Scudder, J., and Sonnerup, B.: First multispacecraft ion measurements in and near the Earth's magnetosphere with the identical Cluster ion spectrometry (CIS) experiment, Ann. Geophys., 19, 1303-1354, 2001.

Seki, K., Hirahara, M., Terasawa, T., Mukai, T., Saito, Y., Machida, S., Yamamoto, T., and Kokubun, S.: Statistical properties and possible supply mechanisms of tailward cold $\mathrm{O}^{+}$beams in the lobe/mantle regions, J. Geophys. Res., 103, 4477-4489, 1998.

Seki, K., Elphic, R. C., Hirahara, M., Terasawa, T., and Mukai, T.: On atmospheric loss of oxygen ions from Earth through magnetospheric processes, Science, 291, 1939-1941, 2001.

Shelley, E., Johnson, R. G., and Sharp, R. D.: Satellite observations of energetic heavy ions during a geomagnetic storm, J. Geophys. Res., 77, 6104, 1972.

Valek, P. W., Perez, J. D., Jahn, J.-M., Pollock, C. J., Wüest, Friedel, R. H. W., Moore, T. E., and Peterson, W. K.: Outflow from the ionosphere in the vicinity of the cusp, J. Geophys. Res., 107, 10.1029/2001JA000107, 2002.

Winglee, R. M., Menietti, J. D., Peterson, W. K., Burch, J. L., Waite Jr, J. H., and Giles, B.: Magnetosheath-ionospheric plasma interactions in the cusp/cleft, 1. Observations of modulated injections and upwelling ion fluxes, J. Geophys. Res., 98, 1931519329, 1993.

Winglee, R. M., Chua, D., Brittnacher, M., Parks, G. K., and Lu, G.: Global impact of ionospheric outflows on the dynamics of the magnetosphere and cross-polar cap potential, J. Geophys. Res., 107, doi:10.1029/2001JA000214, 2002.

Yau, A. W. and André, M.: Sources of ion outflow in the high latitude ionosphere, Space Sci. Rev., 80, 1-25, 1997. 